\title{
eJRIEPS
}

Ejournal de la recherche sur l'intervention en éducation physique et sport

14 | 2008

Varia

\section{Sens des mises en scène didactiques pour le débutant en Judo dans la formation initiale en STAPS}

Éric Margnes, Denis Loizon et André Terrisse

\section{OpenEdition}

Édition électronique

URL : https://journals.openedition.org/ejrieps/5919

DOI : 10.4000/ejrieps.5919

ISSN : 2105-0821

Éditeur

ELLIADD

Référence électronique

Éric Margnes, Denis Loizon et André Terrisse, «Sens des mises en scène didactiques pour le débutant en Judo dans la formation initiale en STAPS », eJRIEPS [En ligne], 14 | 2008, mis en ligne le 01 avril 2008, consulté le 18 juin 2021. URL : http://journals.openedition.org/ejrieps/5919 ; DOI : https:// doi.org/10.4000/ejrieps.5919

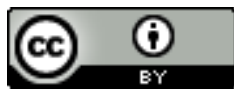

La revue eJRIEPS est mise à disposition selon les termes de la Creative Commons Attribution 4.0 International License. 
Sens des mises en scène didactiques pour le débutant en Judo dans la formation initiale en STAPS

Éric Margnes, Denis Loizon \& Terrisse André

Équipe AP3E, LEMME (EA 3042), Université Paul Sabatier, Toulouse, France

\section{Résumé}

Le but de cette recherche est de montrer que l'on peut inférer l'intention didactique d'un enseignant à partir de l'analyse de son intervention. Le choix des objets d'enseignement et des situations didactiques semble déterminé, à la fois par des conceptions de l'activité judo et par des conceptions de son enseignement à des débutants. La méthodologie utilisée repose sur une analyse qualitative de l'action de deux formateurs de judo en STAPS. L'exploitation des données (observations et entretiens) fait apparaître un jeu complexe entre les diverses conceptions établies et met en évidence le poids des conventions de rôles et de réalisation dans différents types de situations répertoriées. Les différentes mises en scène proposées renvoient à des valeurs, à des normes, bref, à une éthique de la pratique qui détermine un pacte de pratique et un lien social entre les pratiquants.

L'intervention est considérée comme « un acte professionnel mettant en œuvre des compétences, des savoirs d'expérience et théoriques au service d'un objectif » (Lecomte, Carlier, 2000). Nous avons choisi de travailler autour de la problématique de l'intention didactique de l'enseignant en mettant l'accent sur ses déterminations culturelles voire éthiques. Nous postulons l'existence d'une dimension intentionnelle et sensible, en arrièreplan de toute intervention didactique, qui permet de faire émerger du sens à l'activité enseignée (Margnes, 2002). Cette optique renvoie l'enseignant à l'exercice d'une responsabilité (Martinand, 1986 ; Develay, 1992) et à un rapport personnel au savoir à enseigner (Chevallard, 1991). Selon Portugais (1998), l'intentionnalité d'un formateur représente ses croyances, ses désirs, ses volontés et ses intentions. Si elle peut s'exprimer, en partie, dans des entretiens ou des écrits, elle est surtout révélée par des actes, par des jugements en situation de cours, par une manière d'animer les savoirs ; c'est ce que nous entendrons sous l'expression de mises en scène didactiques (Amade- 
Escot, 1998). Dans ce contexte, l'enseignant influence le parcours de formation de l'élève en lui communiquant nombre de ses conceptions.

Cette recherche descriptive et compréhensive étudie l'épistémologie des savoirs puisqu'elle se propose d'expliciter l'origine des savoirs et leur nature. Elle renvoie également à une didactique à dominante anthropologique puisqu'elle s'intéresse à la façon dont certains exercices traditionnels peuvent devenir de véritables savoirs à enseigner (Perrenoud, 1995). La description s'associe ainsi à une perspective herméneutique puisqu'il s'agit de cerner, par l'intermédiaire des mises en scène didactiques, la signification de ce qui est transmis.

\section{La dimension sensible et intentionnelle des savoirs}

1. 1. La mise en scène des savoirs et leur dimension sensible

Le savoir n'a de sens, de valeur, qu'en référence aux rapports qu'il suppose et produit avec le monde, avec les autres et avec soi-même (Charlot, 1997). Le judoka débutant construit son rapport au savoir dans sa relation à l'enseignant, avec ses adversaires, autour des situations didactiques éprouvées et en fonction de ses connaissances antérieures. II acquiert ainsi des compétences motrices et sociales qui s'inscrivent dans un réseau de significations (Peyré, 2000). Les mises en scène didactiques, proposées aux élèves, donnent ainsi une certaine saveur aux savoirs en fixant des modalités d'action et des modalités d'interaction sociale. De ce fait, elles sont les dépositaires de normes et de valeurs à respecter. La recherche présentée s'intéresse donc autant à la mise en situation du savoir qu'il s'agit de transmettre qu'à l'objet de savoir lui-même.

Pour ne pas nous restreindre à un aspect trop rationnel (au sens strict, lié au seul raisonnement), dans cette étude portant sur l'intention didactique, nous avons tenu à aborder une notion «plus intime », celle de la dimension sensible des savoirs. Dans le domaine des pratiques physiques, la variété des expériences corporelles, impose d'établir un lien entre un aspect rationnel de la conduite (des buts à attendre) et un aspect plus sensible, propre au vécu, au ressenti de l'acteur. Griffet (1996) évoque à ce sujet le poids de la dimension sensible fondamentale et celui de la construction symbolique de la réalité... Cet aspect rejoint une forme d'esthétique, au sens de Maffesoli (1990) ; c'est une forme de sensualisme quotidien qui exprime une « raison sensible », dans laquelle le plaisir des sens, le jeu des formes, le retour en force de la nature, l'intrusion du futile, appellent à une connaissance plus ouverte, à une « hyper rationalité ». L'introduction de cette part de subjectivité dans l'activité est d'inspiration phénoménologique (MerleauPonty, 1945) ; elle affirme la dimension vécue du corps. L'intentionnalité des enseignants 
doit ainsi être envisagée comme une résultante de comportements rationnels et de rapports plus intimes éprouvés en tant que pratiquant.

\section{2. De l'intentionnalité à l'intention didactique}

Portugais (1998) opère une distinction entre l'intentio (le projet social d'enseignement caractérisant les objets de savoir et leur sens), l'intentionnalité (l'intention privée de l'enseignant qui met en marche les positions intentionnelles) et les intentions didactiques qui sont mises en jeu dans une situation donnée pour déterminer un bon fonctionnement des problèmes à résoudre. Si certaines intentions sont des décisions prises sur le vif, d'autres sont choisies pour parfaire la négociation du contrat didactique (Brousseau, 1996) ou pour permettre le contrôle social des apprentissages (Chevallard, 1991).

Dans ce cadre conceptuel, le processus de transposition didactique (Verret, 1975 ; Martinand, 1986 ; Chevallard, 1991), est au fondement de l'intention didactique puisqu'il permet d'étudier le décalage entre l'intentio, l'intentionnalité et les intentions didactiques. Les caractéristiques propres au processus de transposition didactique (Martinand, 1986) ont ainsi servi de cadre d'analyse pour examiner l'enseignement du judo dans ses rapports à la référence sociale, dans ses modes d'intervention et dans ses modalités de construction du sens de la pratique. Le contrat didactique (Brousseau, 1986) devient la cheville ouvrière de l'intentionnalité et par voie de conséquence des intentions didactiques, en situation.

Outre la hiérarchisation des objets d'enseignement et leur mise en problème, l'analyse des conventions, définies comme l'ensemble des règles explicites voire tacites qui déterminent un accord, un pacte de pratique entre les différents protagonistes, a retenu notre attention. Ces conventions nous semblent être un des moyens privilégiés pour déclencher, chez l'apprenant, la compréhension mais surtout l'adhésion à une conception de l'activité (Schubauer-Léoni, 1996).

1. 3. L'intentionnalité : des choix culturels d'ordre éthique et technique

De nombreux auteurs ont exposé combien la notion de technique ne pouvait se restreindre à la simple efficacité gestuelle et qu'il convenait de la replacer dans différents contextes ou systèmes (tendance technique chez Leroi Gourhan (1973) ; pensée technique chez Picon (1994). Selon Deforges (1993), l'enseignement des techniques est culturel et les techniques sont d'abord gouvernées par une éthique. L'activité technique intègre ainsi, dans la logique du combat de judo, toutes les manières de faire pour créer et utiliser les forces nécessaires au combat debout et au sol, mais aussi les façons de se comporter. Ces manières de faire renvoient inévitablement à une représentation de l'efficacité, à des valeurs, des normes, à une éthique de la pratique. Dans une autre étude 
sur l'enseignement du judo, Loizon (2004) a pu montrer le poids des déterminants personnels liés à l'expérience de judoka. Russ (1994) définit l'éthique comme « une doctrine au-delà de la morale » qui permet de « déconstruire les règles de conduite pour s'efforcer de descendre jusqu'aux fondements cachés de l'obligation ». Elle est, selon Habermas (1987), indissociablement praxis et réflexion et est fondée à partir du concept de rationalité qui s'attribue selon « sa capacité d'être critiquée et fondée ». Cette rationalité peut être de nature téléologique (sens utilitariste) ou axiologique (normes éthiques).

L'analyse préalable de cette recherche a débuté par une revue de la littérature portant sur l'histoire du judo, son enseignement à des débutants, tant dans la sphère fédérale que dans le cadre de l'EPS. Elle a permis de mettre en évidence différentes logiques de rationalité (critères portant sur les valeurs éducatives de l'activité, sur le sens accordé au combat, sur la valeur des actes) et surtout d'établir un cadre d'analyse des différentes conceptions du judo.

Nous avons également relevé d'autres conceptions touchant, cette fois-ci, à l'enseignement de l'activité (Marsenach, 1991) qui nous ont permis de distinguer une approche formelle et une approche fonctionnelle.

- L'approche formelle attribue à la forme extérieure des actes une portée décisive (Brousse, 1994) ; elle conduit l'enseignant de judo à accorder une dimension formatrice à la technique, en privilégiant autant la démonstration que des situations en partenariat, hors du contexte de l'opposition.

- L'approche fonctionnelle met l'accent sur l'investissement des élèves dans des situations d'affrontement authentiques avec cadre réglementaire minimal (Barbot, 1996) ; il s'agit de situations en opposition permettant à l'élève d'expérimenter des solutions et de s'éprouver dans la confrontation aux autres.

Tableau I. Les deux conceptions de l'activité judo

\begin{tabular}{|c|l|l|}
\hline Conceptions de l'activité & \multicolumn{1}{|c|}{ Judo traditionnel } & \multicolumn{1}{c|}{ Judo sportif } \\
\hline Valeur de l'activité & $\begin{array}{l}\text { Épanouissement personnel par } \\
\text { l'entraide. }\end{array}$ & $\begin{array}{l}\text { Valorisation de soi par la } \\
\text { hiérarchisation avec autrui. }\end{array}$ \\
\hline Sens du combat & $\begin{array}{l}\text { Progrès personnel au service } \\
\text { d'une utilisation optimale de } \\
\text { l'énergie, d'une esthétique alliée } \\
\text { à l'efficacité. }\end{array}$ & $\begin{array}{l}\text { Progrès personnel au service } \\
\text { du résultat compétitif, de la } \\
\text { rentabilité technique. }\end{array}$ \\
\hline Valeur des actes & $\begin{array}{l}\text { Recherche d'un idéal de forme } \\
\text { et d'un idéal de « force - } \\
\text { souplesse »dans engagement } \\
\text { total dans l'action. }\end{array}$ & $\begin{array}{l}\text { Utilisation de tous les moyens } \\
\text { physiques, techniques, } \\
\text { stratégiques adaptés au } \\
\text { règlement. }\end{array}$ \\
\hline
\end{tabular}




\section{La problématique de recherche}

La compréhension d'une intention didactique s'incarne dans des stratégies d'intervention et des mises en scène qui témoignent des prises de position de l'enseignant dans le champ culturel et social. Le choix des objets d'enseignement, celui des situations didactiques - leurs mises en scène, leurs mises en valeur - semblent déterminés :

- d'une part, par deux conceptions possibles de l'activité judo : un judo traditionnel et un judo sportif ;

- d'autre part, par deux façons de concevoir l'enseignement du judo à des débutants : une conception fonctionnelle, liée à une vision instrumentaliste de la technique, et une conception formelle, intégrant une vision formatrice de la technique.

\section{La méthodologie de recherche qualitative}

Notre travail s'inscrit dans le cadre d'une recherche qualitative, telle que l'ont développée différents auteurs. (Deslauriers, 1987 ; Mucchielli, 1991 ; Van Der Maren, 1996). Cette recherche comprend aussi une "posture clinique »(Revault d'Allonnes, 1999) puisque nous travaillons à partir d'études de cas de deux enseignants intervenant en formation initiale en STAPS (Sciences et Techniques des Activités Physiques et Sportives). En fait, nous cherchons à explorer le rapport singulier et personnel qu'entretiennent ces deux experts à l'activité qu'ils ont choisi d'enseigner (Terrisse, 2000).

Nous avons aussi dû imaginer une procédure de recueil des données et d'analyse pour réduire les données observées, les condenser en fonction des axes de réflexion privilégiés (Huberman, Miles, 1983). Des questions - guides portant sur la transposition didactique (objet de travail, rôles et conventions proposés, problèmes soulevés, modes d'intervention, savoirs produits) ont joué un rôle déterminant dans la conduite de l'analyse, notamment des entretiens. Nous avons souhaité opérer une triangulation des données pour combiner plusieurs techniques de recueil afin de compenser le biais inhérent à chacune d'elles (Savoie-Zajq, 1996). Elle permet d'apprécier la richesse et la complexité des phénomènes humains étudiés.

Ont été menés successivement :

- Un entretien initial dont la fonction est de saisir une conception, a priori, de l'enseignement du judo à des débutants, « une intentionnalité avancée » ; cet entretien de type semi dirigé a permis de préserver une certaine liberté d'expression tout en orientant l'interview. 
- Une observation directe dans le vif de la séance (prise de notes à la volée) et indirecte (relecture et retranscription des enregistrements audio et vidéo) de l'intervention de chaque enseignant durant huit séances.

- Un entretien à l'issue du cycle de formation. Cette étape de «l'après-coup » est essentielle car elle permet à l'acteur de revenir sur son action pour éviter au chercheur les contresens et les interprétations abusives. Cet entretien d'auto confrontation à des évènements marquants (relevés par le chercheur à l'appui des discours retranscrits ou de la vidéo) a permis aux enseignants de mieux expliciter certaines prises de décisions et de recentrer les enseignants sur nos interrogations de recherche.

De nombreux auteurs ont ainsi souligné la difficulté de l'observation de la pratique d'intervention dont l'enjeu est de faire apparaître un réseau de significations en relation avec l'objet d'étude (Postic, De Ketele, 1988). Nous avons souhaité respecter un certain nombre d'étapes, inspirées par la recherche des formes de situation (Mucchielli, 1991), en répertoriant différents types de situations et en analysant leurs modalités de mise en scène et de mise en valeur. Nous avons d'abord distingué des catégories de situations communes à tout intervenant (prise en charge du groupe, situations d'échauffement, situations didactiques au sol et debout, de présentation et de régulation). Si chaque type de situation est porteur de signification, nous avons fait le choix, dans le cadre de cet article, de développer essentiellement les situations didactiques d'étude ; en effet, elles soulignent l'importance des conventions de rôles à tenir (façon d'être et de se comporter avec autrui) et de réalisation des actes (façon d'exploiter les énergies, les possibilités offensives et défensives et d'obtenir un résultat) qui permettent d'inférer des conceptions. Nous avons répertorié différents types de situations d'étude :

- Des situations sans opposition (série 1, couleur bleue). Le partenariat permet d'appréhender les rôles de tori (celui qui agit) et de uke (celui qui subit).

- Des situations avec une opposition modulée et orientée (série 2, couleur rouge) ; le judoka est, soit attaquant, soit défenseur ; pour chaque rôle, l'opposition est plus ou moins forte, les possibilités d'action plus ou moins définies.

- Des situations de combat (série 3, couleur jaune) : le judoka est dans la complexité d'une opposition conventionnelle, en référence au judo sportif ou au judo traditionnel.

Dans un deuxième temps, nous avons ainsi comptabilisé le nombre total de type de situations proposées par les enseignants aux étudiants, lors de huit séances successives ; ensuite, nous avons recherché des éléments analogues et distincts dans chaque catégorie de situations, les éléments distincts permettant de singulariser l'intervention de chaque formateur. 


\section{Les résultats}

Un premier bilan quantitatif portant sur les types de situations employées par chaque enseignant permet de comparer les deux interventions pour le judo debout et pour le judo au sol (figure 1 ci-dessous). Ainsi, sur l'axe des abscisses, les zones 1 et 2 correspondent aux séries de situations du formateur 1 (F1) pour le judo debout et pour le judo au sol ; les zones 5 et 6 concernent les séries de situations du formateur 2 (F2), pour le judo debout et le judo au sol. On visualise d'emblée combien les interventions se différencient, notamment pour le judo debout, quant à l'emploi des différents types de situation ; ainsi, le formateur 2 utilise près de deux fois plus de situations sans opposition que le formateur 1 (18 situations série 1 contre 10 pour l'autre) et près de deux fois moins de situations de combat (2 situations série 3 contre 4 pour le formateur 1 ).

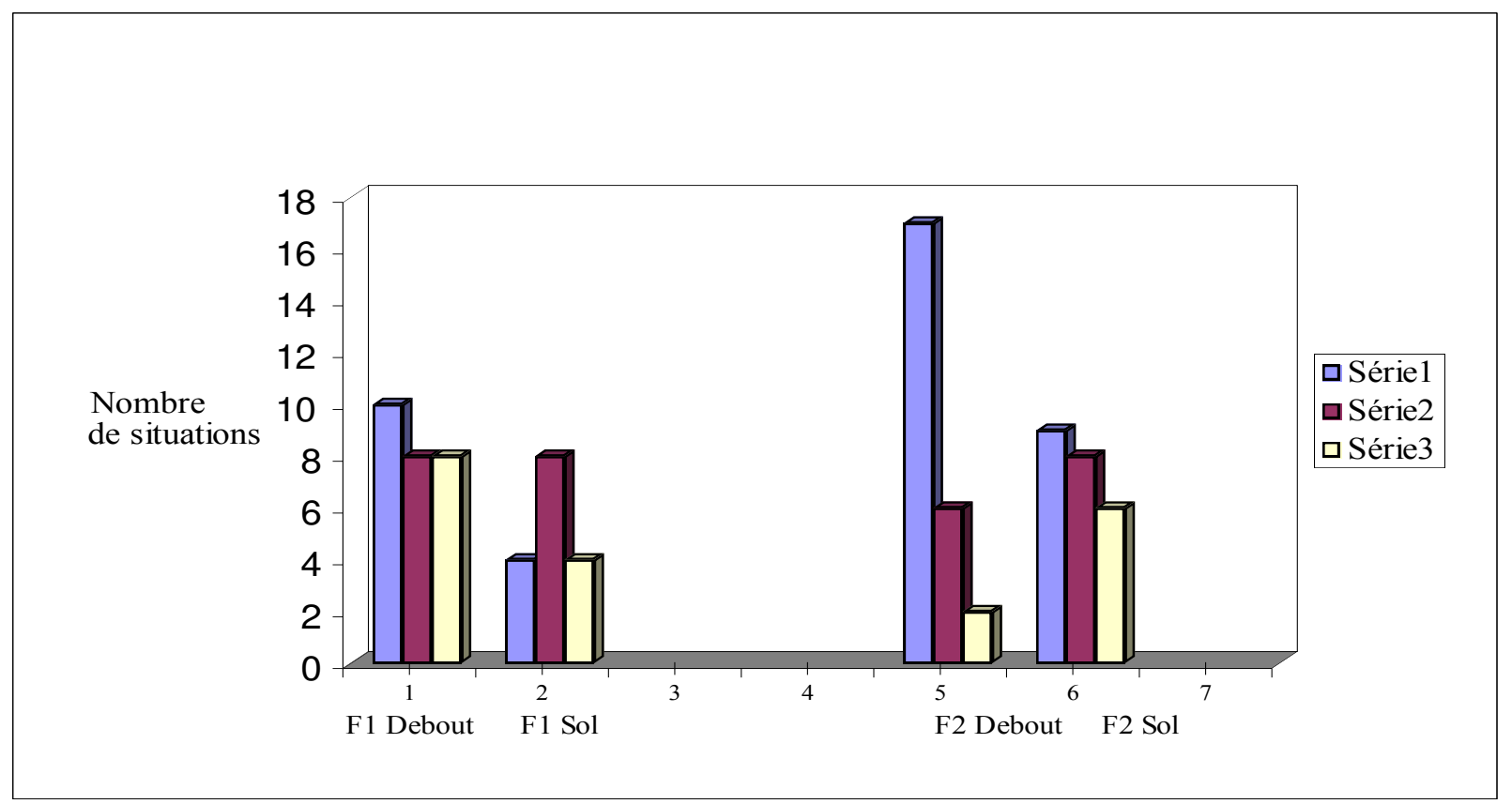

Figure 1. Comparaison quantitative des situations utilisées par chaque formateur

L'analyse qualitative de l'intervention repose sur la mise en relation des différents modes de recueil de données. Nos inférences se sont appuyées sur un relevé de conventions tiré des observations des séances et sur des morceaux choisis, extraits des entretiens. Nous donnons, ci-après, un extrait d'un entretien d'après-coup qui permet au formateur de revenir sur son expérience vécue, d'approfondir la signification de certains gestes, expressions ou attitudes relevées en situation. Le chercheur peut ainsi confirmer ou non les inférences tirées du constat des faits.

L'entretien d'après-coup de F1 (morceaux choisis) - Question relative aux expressions de « justesse technique, d'invention de technique, de perfection des actes », utilisées par F1 : «L'esthétique dans l'action, pour moi, est présente, mais doit inclure un nécessaire rapport d'opposition. Cela me gêne de voir dans l'esthétique, une production de forme 
répétée dans la seule intention de montrer une esthétique. L'image extérieure renvoie de l'esthétique et la perfection relève de la perception intime dans l'opposition. Moi j'ai envie que le judoka, qui a fait la forme, ne l'ait pas faite, à priori, mais élaborée en situation duelle. (...).C'est vrai, au sol, j'ai l'impression que la dynamique de l'accrochage restreint cette esthétique mais engage le judoka dans une rigueur des pré-requis efficaces ; l'aspect tactique réfère à un accrochage initial ».

Le caractère volontairement descriptif du mode et de l'objet de l'intervention de chaque formateur expose à quelles conceptions préalablement identifiées le chercheur attribue les constats effectués.

\section{1. Le mode d'intervention}

Le formateur 1 a une prédilection pour les situations en opposition, notamment en opposition orientée (un attaquant, un défenseur). Les situations sans opposition sont peu utilisées (elles permettent à l'enseignant de reconnaître parfois un savoir découvert par certains étudiants). II se dégage de l'entretien d'après-coup que cet enseignant ne souhaite pas démontrer de formes techniques à reproduire, ni mettre l'accent sur un aspect esthétique de la technique : il parle de « jeu avec l'autre, de rapport constructif ». Debout comme au sol, il insiste sur une activité fonctionnelle dans laquelle les étudiants doivent chercher des solutions à la fois techniques et tactiques. II ne nomme que très rarement les techniques en japonais et préfère parler en termes de principe d'efficacité, de repères : « la proximité du partenaire sera essentielle car c'est elle qui permet de produire de la force ; mon bassin doit être collé et sous le bassin de l'adversaire ». Cette activité fonctionnelle est cependant «encadrée » par de nombreuses consignes relatives à la sécurité, notamment pour le judo debout : « ne pas tomber sur l'autre, ne pas aller au sol pour projeter ».

A l'inverse, le formateur 2 part souvent de sa démonstration personnelle pour mettre les élèves en activité. Le travail important de reproduction technique, en partenariat, a pour objet de faire sentir la technique et d'éprouver une certaine relation à l'autre : « la bonne ou la mauvaise exécution vient du partenaire ". La notion de forme de corps occupe surtout une place centrale dans l'approche du judo debout: «j'ai pris le raccourci de la démonstration pour cibler tout de suite l'aspect technique de l'activité ». Les techniques transmises sont ensuite testées dans des situations en opposition orientée. L'intervention repose sur différents types de solutions apportées par l'enseignant: par présentation de formes de corps (démontrées et nommées en japonais) : « II y a deux grands types de contrôle : les shio gatamé qui sont des contrôles poitrine-poitrine et les gésa gatamé qui sont des contrôles latéraux. Je vais montrer quelques immobilisations faciles », par 
évocation de principes tactiques (sous la forme d'enchaînements prédéterminés) et parfois même par anticipation de problèmes à venir (certains objets d'enseignement, propres à différents types de saisies, ont été proposés en réponse à des comportements défensifs éventuels et non en rapport à l'activité réelle des étudiants). Au sol, nous relevons un travail en opposition conventionnelle : «On va faire du randori au sol (combat d'entraînement valorisant l'offensive). On va chercher à immobiliser dix secondes. On va s'arbitrer. A partir de là, on va enchaîner une autre immobilisation ».

\section{2. L'objet de l'intervention}

Le formateur 1 donne la priorité au travail debout par rapport au travail au sol et met en avant les aspects tactiques ; ainsi, l'entrée dans l'activité s'est effectuée à partir du jeu de sumo. Debout, sont privilégiées les notions d'initiative et de continuité des actions à partir d'un combat sécurisé. Le rapport au règlement est fort; la saisie lors de l'attaque est codifiée : «contrôler le bras contrôlé du côté de l'attaque » et les conventions sur les modalités offensives et défensives sont contraignantes : « ne pas tomber sur l'autre, ne pas aller au sol pour projeter, esquiver pour défendre, ne pas tendre les bras ». Debout, l'activité de référence oscille donc entre un judo traditionnel, traité sous le versant de la maîtrise et de la sécurité et un judo sportif traité sur le versant du gain du combat, de positions dominantes alors qu'au sol, la logique sportive est ainsi bien plus présente.

Le formateur 2 a souhaité entrer dans l'activité par le judo au sol propédeutique, selon lui, au travail debout. II divulgue de nombreux contenus techniques qui intègrent des aspects spécifiques à chaque forme de corps et des principes d'efficacité communs : « la création du contact du haut du corps pour projeter ». Au sol, il propose une confrontation intégrant une forme de collaboration car il demande aux défenseurs d'éviter les positions négatives: «ne pas rester à plat ventre » et aux attaquants d'enchaîner une immobilisation quand un premier point est marqué. Debout, il a très rapidement abandonné la notion de blocage comme modalité défensive pour privilégier l'esquive et a insisté sur la variabilité des attaques qui imposent à uke de s'habituer à chuter dans des circonstances diverses : « on peut attaquer sans le contrôle du bras du côté de l'attaque ». La responsabilité tacite de la sécurité incombe donc autant au respect d'une « bonne forme de corps » par l'attaquant qu'à un bon esprit de coopération pour uke : «accepter de chuter ». Debout, la référence de cet enseignant semble plus proche d'un judo traditionnel dans laquelle il importe de montrer une «bonne forme de corps », en s'engageant pleinement dans l'action, au-delà du résultat du combat. Au sol, la référence à l'une ou l'autre des conceptions est moins marquée. 


\section{Discussion}

Tout au long de cette recherche, nous avons eu le souci de montrer combien l'intentionnalité d'un enseignant est tributaire d'un rapport personnel à l'activité enseignée et d'une conception de son enseignement à des débutants. Si les résultats ont confirmé notre modèle initial, ils ont également permis d'en éclairer certains mécanismes ou principes fonctionnels :

Pour l'approche du combat debout, nous avons relevé deux possibilités :

- L'optique d'un judo traditionnel, se référant à une performance maîtrisée avec la conquête de la verticalité descendante dans la mise à terre de l'adversaire (Jeu, 1977) ; cette conception met l'accent sur la notion d'idéal de forme et sur un rapport d'entraide entre les partenaires.

- L'optique d'un judo sportif, associé à un souci sécuritaire, dans lequel on valorise une activité fonctionnelle régie par la règle ; cette conception met l'accent sur le jeu, l'initiative, la domination, le résultat, tout en conservant un rapport constructif à l'autre.

L'approche du judo au sol prédispose plus à la conception d'un judo sportif, avec le plein engagement physique propre au maintien de l'immobilisation. Cependant, la suppression des clés et des étranglements, adoptée par les deux formateurs, ôte cette dimension de performance maîtrisée propre au judo traditionnel. La modélisation suivante (figure 2) permet de situer l'intervention de chaque formateur (F1 et F2).

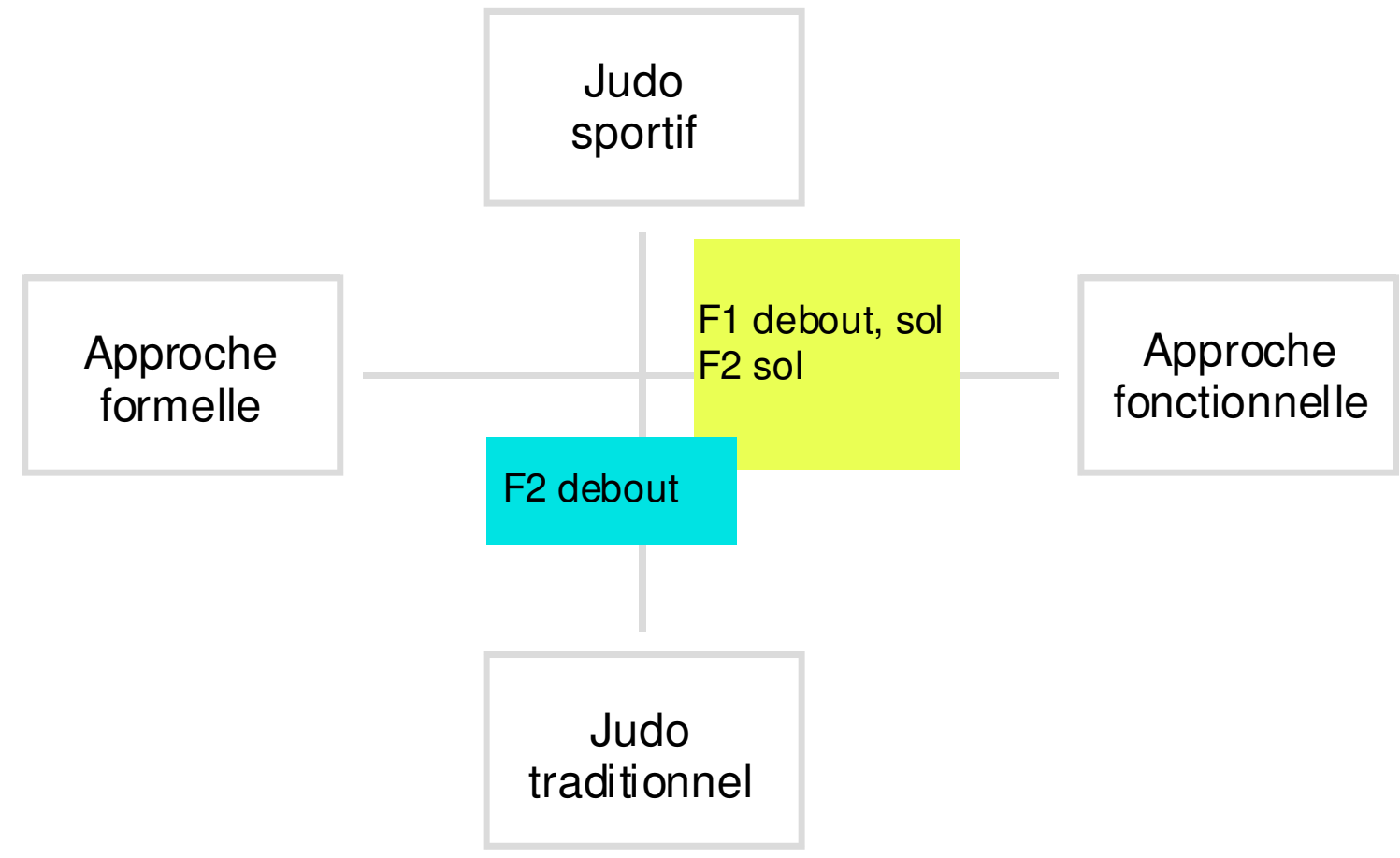

Figure 2. Intervention et intentions de formation 
Dans l'approche formelle du judo sportif (secteur 1 du schéma), la transmission de formes est issue de l'observation du haut niveau ou des solutions trouvées par les étudiants. Le geste technique est utilisé dans le versant utilitariste de l'efficacité combative. Dans l'approche formelle du judo traditionnel (secteur 4), le travail de la forme de corps permet la recherche de la perfection dans l'acte, la sensation de la juste utilisation de l'énergie adverse et éventuellement l'accès à une dimension expressive. C'est un geste chargé en « valeur ajoutée ».

Dans la perspective fonctionnelle sportive (secteur 2), l'intervenant fait confiance à la règle pour garantir la sécurité dans les actions entreprises (F1 et F2 pour le judo au sol). Dans l'approche fonctionnelle traditionnelle (secteur 3), l'étudiant est amené à résoudre des problèmes posés par l'adversaire, mais dans le respect de règles éthiques. Un mouvement mal porté, un mouvement qui «force » est jugé non pas comme un échec dans la domination de l'adversaire, mais comme un échec personnel au niveau d'une certaine idée de la force et de la relation à l'autre (F2, pour le judo debout).

\section{Conclusion}

L'observation des pratiques de formation a fait apparaître que les conceptions, identifiées dans l'analyse préalable, ne sont pas aussi tranchées que nous aurions pu l'imaginer. D'une part, la variabilité de l'intention fait qu'un enseignant peut passer d'une conception de l'activité à une autre, en insistant sur de « bonnes formes de corps traditionnelles » et en proposant différentes saisies issues du judo compétitif. D'autre part, la séparation artificielle du judo au sol et du judo debout, effectuées par les deux formateurs, a eu des effets non prévus. Elle a permis, aux deux enseignants, de véhiculer conjointement les deux conceptions de l'activité (une, pour le judo au sol, l'autre, pour le judo debout).

En fait, l'intentionnalité d'un formateur est surtout révélée par une manière d'animer les savoirs et de les mettre en valeur. Les études de cas ont permis de révéler une part des mécanismes qui la déterminent grâce à l'analyse des conventions. Elles ont souligné les rapports singuliers et complexes existants entre un formateur et une activité enseignée. Cette recherche qualitative n'a pas l'ambition de rendre compte de tous les types d'intervention possibles mais d'exposer la pertinence d'un modèle des pratiques de formation en judo croisant des conceptions de l'activité avec des conceptions de son enseignement. 


\section{Bibliographie}

Amade-Escot, C. (1998). Apport des recherches didactiques à l'analyse de l'enseignement: une étude de cas, le contrat didactique. In C. Amade-Escot et al.(Éds.), Recherches en EPS : Bilan et perspectives (pp. 253-266). Paris : Revue EPS.

Barbot, A. (1996). Approche technologique des phénomènes d'enseignement du Judo en EPS. Thèse non publiée, Université Paris XI. Orsay.

Brousse, M. (1994). La technique ou l'ordre du combat. In Dossier EPS $n^{\circ} 19$, Techniques sportives et EPS (pp. 80-89). Paris : Revue EPS.

Brousseau, G. (1986). Théorisation des phénomènes d'enseignement des mathématiques. Thèse d'Etat, Université de Bordeaux I.

Charlot, B. (1997). Du rapport au savoir : Éléments pour une théorie. Paris : Anthropos.

Chevallard, Y. (1991). La transposition didactique, du savoir savant au savoir enseigné. Grenoble : La Pensée Sauvage.

Deforges, Y. (1993). De l'éducation technologique à la culture technique. Paris : ESF.

Deslauriers; J.P. (1987). Les méthodes de la recherche qualitative. Sillery: Presses Universitaires du Québec.

Develay, M. (1992). De l'apprentissage à l'enseignement. Paris : ESF.

Griffet, J. (1996). Pratiques corporelles libres et transmission des savoirs. Impulsions, 2, 65-73, INRP .

Habermas, A. (1987). Théorie de l'agir communicationnel. Paris : Fayard.

Huberman, M., \& Miles, M. (1983). L'analyse des données qualitatives. Cahier du GCR/SSRE,6. Neufchâtel : Institut Romand de recherches et de documentations pédagogiques.

Jeu, B. (1977). Le sport, l'émotion, l'espace. Paris : Vigot.

Lecomte, J. \& Carlier, G. (2000). Intervenir en éducation physique. In G. Carlier (Éd.), Encadrer des stagiaires en éducation physique (pp. 13-23). Louvain La Neuve : UCL EDPM.

Leroi Gourhan, A. (1973). Milieu et Techniques. Paris : Albin Michel.

Loizon, D., Margnes, E., Terrisse, A. (2004). Analyse des pratiques d'enseignement : les pratiques déclarées par les professeurs de judo, eJRIEPS, 5. IUFM de Besançon.

Maffesoli, M. (1990). Au creux des apparences, Pour une éthique de l'esthétique. Paris: Plon. 
Margnes, E. (2002). L'intention didactique dans l'enseignement du judo, des choix culturels d'ordre éthiques et techniques. Etude de cas de situations didactiques leurs mises en scène - pour des débutants dans la formation initiale en STAPS. Thèse non publiée. Université de Toulouse III.

Marsenach, J. (1991). Éducation Physique et Sportive. Quel enseignement ? Paris : INRP. Martinand, J.L. (1986). Connaître et transformer la matière. Berne : Editions Peter Lang. Merleau-Ponty, M. (1945). Phénoménologie de la perception. Paris : Gallimard.

Mucchielli, A. (1991). Les méthodes qualitatives. Que sais-je ? Paris : PUF.

Perrenoud, P. (1995). Enseigner des savoirs ou développer des compétences : l'école entre deux paradigmes. In A. Bentolila (Éd.), Savoirs et savoir-faire (pp. 73-88). Paris : Nathan.

Peyre, P. (2000). Compétences sociales et relations à autrui. Une approche complexe. Paris : L'harmattan.

Picon, A. (1994). Le dynamisme des techniques. In R. Scheps (Éd.), L'empire des techniques (pp. 25-35). Editions du Seuil.

Portugais, J. (1998). Esquisse d'un modèle des intentions didactiques, Contribution à la didactique des mathématiques. In J. Brun, F. Conne, R. Floris \& ML SchubauerLeoni (Éds), Interactions didactiques, Méthodes d'étude du travail de l'enseignant (pp. 57-88). Genève : Actes des secondes journées didactiques de la Fouly.

Postic, M. \& De Ketele, J.M. (1988). Observer les situations éducatives. Paris : PUF. Revault d'Allones, C. (1999). La démarche clinique en Sciences humaines. Paris : Dunod. Russ, J. (1994). La pensée éthique contemporaine. Que sais-je ? Paris : PUF.

Savoie-Zajq, L. (1996). Triangulation. In A. Mucchielli (Éd.), Dictionnaire des méthodes qualitatives en sciences humaines et sociales. Paris : Armand Colin.

Schubauer-Léoni (1996). Etude du contrat didactique pour les élèves en difficulté en mathématiques. In C. Raisky \& M. Caillot (Éds), Au-delà des didactiques, le didactique (pp. 160-189). Bruxelles : De Boeck, 1996.

Terrisse, A. (2000). Epistémologie de la recherche clinique en sport de combat. In Terrisse (Éd.), Recherches en sports de combat et en arts martiaux (pp. 95-108). Paris : Editions Revue EPS.

Van Der Maren, J.-M. (1996). Méthodes de recherche pour l'éducation. Bruxelles: De Boeck.

Verret, M. (1975). Le temps des études. Paris : Honoré Champion. 